\title{
Analysis of Military Application of Software Radio Communication Technology
}

\author{
Jing Gao \\ Operation Software and Simulation Research Institute of Dalian Naval Academy, Dalian 116018, China
}

\begin{abstract}
The emergence of software radio technology is the third technological revolution in the field of communication. In view of the immature status of the application of software radio communication technology in our country's military affairs, on the premise of interpreting the connotation and extension of software radio communication technology, this paper systematically analyses the principle and architecture of software radio, and puts forward that military software radio communication technology can be used in electronic warfare simulation evaluation and software enhancement. The conception of the application of wire electronic warfare system expands the theoretical breadth of military application of software radio communication technology.
\end{abstract}

\section{The connotation and extension of software radio communication technology}

The so-called software radio communication technology refers to the radio communication equipment which has the characteristics of realizing various functions by software, and the radio communication function is completed by software design, which is a completely programmable ability realization. Including alterable channel access mode, channel modulation mode and error correction algorithm, it completely gets rid of the traditional use-oriented design idea. The important value of software radio technology lies in that the traditional hardware radio communication equipment is only the basic platform of wireless communication, and many communication functions are realized by software, which breaks the pattern that the realization of communication functions of equipment only depends on the development of hardware. At the same time, through the reprogramming of software radio, the system can be updated and upgraded to adapt to different communication standards. Wireless communication system can be upgraded and used by software upgrade in the process of using. Usually, the switching of communication system can be carried out according to the standard by redefining the modem process, air wireless interface and decoding and coding method. Its core idea is based on the open, standardized and modular general hardware platform, combined with the objectoriented standardized software specifications, which can change the functional attributes of equipment by changing the way of software, reduce the complexity of equipment, and have superior flexibility, centralization and maintenance. Now it is gradually in use. Modern military electronic equipment has been popularized and applied, and become an important industry standard.

\section{The principle and architecture of software radio}

\subsection{Principle of software radio communication technology}

The key idea of so-called software radio is to use software to accomplish various functions (such as working frequency band, modem type, data format, encryption mode, communication protocol, etc.) on the basis of general hardware platform, so as to realize the high flexibility and scalability of radio electronic equipment system. Specifically, software radio emphasizes software controllability and redefinition. Equipment development units can achieve specific combat functions by calling different software units. Business functions are enhanced by software modules, and software can be continuously upgraded, updated or even replaced. For software radio hardware, due to standards-based. The idea of modular design supports continuous iterative survival. In view of the software characteristics of software radio functions, it supports various communication waveforms and wireless link protocols. It can also be compatible with the old system and equipment, and improves the comprehensive costeffectiveness ratio in terms of equipment life cycle, cost, and maintenance and so on.

\subsection{Architecture of software radio communication technology}

\footnotetext{
${ }^{a}$ Corresponding author: likandl@126.com
} 
Because of the advantages of software radio, the United Tactical Radio System (JTRS) of the U.S. Army adopts the design idea of software radio, defines the software communication architecture (SCA) specification, and has been applied all over the world. Through SCA software platform, hardware layer and waveform function software layer are separated from each other, so that software and hardware have portability, configurability, scalability and reusability, forming a standardized software radio platform.

\section{Application conception of military software radio communication technology}

In the process of using military communication system, compared with civil communication system, it requires higher and more complex technology. Because advanced communication equipment is relatively expensive, much communication equipment is used more in developed countries. At present, the development of military communication equipment in our country is still in its infancy, and the use of technology is still being explored and studied. With the continuous progress of science and technology in our country, great breakthroughs will be made in the construction of communication system in the aspects of anti-laterality, anti-interference and antiinterception. At the same time, great breakthroughs will be made in the construction of communication system. In addition, remarkable progress will be made in the capacity of the communication system and the resilience in the transmission process. With the continuous progress of our country's military, software radio technology will be used in various military fields scientifically and reasonably, and strengthen our military strength.

\subsection{EW simulation evaluation system}

How to maximize the operational effectiveness of EW equipment in actual combat is a very urgent problem to be studied clearly. The usual practice is to establish equipment simulation demonstration system, evaluation system and experimental system for equipment singlepackage demonstration and system application demonstration. There are two main problems: first, there are doubts about data sources and reliability in pure soft system demonstration; second, the traditional combination of hardware and software costs a lot, which is limited by environment, personnel, and venues and so on. Using the system design method of software radio, the above problems can be solved by generating and receiving data based on software radio technology. Based on the original EW equipment evaluation and experimental system, software radio technology is introduced to simulate the underlying equipment. Because of the flexibility of software radio, it can simulate the modern battlefield environment more conveniently, quickly and vividly, at the same time, it can send and receive the real data. These data, as the basis of the whole system evaluation and experiment, can solve the problem of low reliability caused by the reliable imitation of the original data.

\subsection{Enhanced software radio electronic warfare system}

The future electronic warfare system should be able to self-adaptive reconnaissance perception of the target and surrounding environment. Based on the large data and deep learning processing of fast and accurate analysis, the optimal electronic warfare strategy should be adaptively selected. Combining with battlefield situation graph, the effectiveness evaluation of electronic warfare can be formed. Finally, the electronic warfare system can be updated according to the evaluation results. Policy resource library. In terms of structure, the closed-loop structure of "observation, positioning, decision-making and action" is mainly adopted. In order to cope with the characteristics of modern electronic warfare, such as changeable signal system and changeable pattern, it is necessary to gradually increase the component of software radio, form the ability of intelligent cognitive electronic warfare, adjust the working parameters of electronic warfare equipment adaptively based on battlefield perceptual learning, and make the electronic warfare system more effective in the future battle in the electromagnetic field. Activity and adaptability. Software radio technology is the first to play a pillar role in electromagnetic spectrum recognition. It focuses on communication, radar and photoelectric signals in complex electromagnetic environment of battlefield. Based on signal processing processes such as detection, measurement, feature extraction, classification and recognition, it combines modern intelligent processing technologies such as large data processing and deep learning. Objectives are positioned cognitively to provide basis for decision-making and action. Software radio technology enhances the hardware universality and software fictionalization of the equipment, which can diversify the objects of investigation, and adjust the reconnaissance strategy in real time to make the reconnaissance equipment more versatile. In decisionmaking and action, software radio technology makes equipment not limited to traditional jamming modes and modes. It can allocate resources and select jamming waveforms by combining knowledge base and jamming strategy resources. It can also evaluate the effect of countermeasures according to the change of object behaviour characteristics after countermeasures, and guide the optimization of countermeasures. Better response to future battlefield needs. The technical characteristics of this enhanced software radio technology equipment are that, on the basis of traditional radio frequency hardware equipment such as receivers, transmitters and antennas, modern software, networking and other technologies are highlighted, advanced algorithms are used to add intelligent functions to electronic warfare, and combined with software radio related frontier technology, radio frequency electronic equipment is equipped with. Stronger survivability. 


\subsection{Software radio radar system}

Taking the design of radio frequency part of radar system as an example and referring to several standardized product structures, software radio technology is applied to intermediate frequency and baseband processing. The radar radio frequency system based on Software Radio fully draws lessons from the programming design idea of software engineering, adopts the open platform design, divides the system function into different module structure, is easy to modify and upgrade the system function, saves the manufacture cost of the radar system, and improves the performance of the radar system. The RF front-end adopts a universal design, which has a wide frequency range and can realize low noise amplification and mixing. In order to realize the maximum software control of radar system function, the IF and baseband signal processing are unified into digital signals for processing, which can better complete the work of frequency conversion, filtering and secondary sampling, further improve the detection sensitivity of radar system, and further enhance the real-time performance of digital signal processing. In addition, the signal modulation of the transmitting system is realized by software control, and various forms of waveforms are generated by programming.

\section{Conclusion}

The application of software radio technology has brought a profound technological change to the field of radio communication, and has played a very good reference and leading role in the technological development of equipment in other fields, especially in the field of electronic countermeasures. It can be predicted that in the near future, with the continuous maturity of software radio technology, it will play a great role in the development, evaluation, testing and practical application of $\mathrm{EW}$ equipment.

\section{References}

1. $\mathrm{Xu}$ Genshen. Introduction to Software Radio [J]. Modern Communication Technology, 1996 (4): 2731.

2. Yang Xiaoniu, Lou Caiyi, Xu Jianliang. Principles and Applications of Software Radio [M]. Beijing: Electronic Industry Press, 2001:227-257.

3. Wang Zhitian. Development analysis of military software radio communication technology [J]. China New Communications, 2016, 18 (24): 23.

4. Li Fei, Suxin, Zeng Jie. Software radio technology and its application in military field $[\mathrm{J}]$. Science and technology and engineering, 2012, 12 (11): 26652672.

5. Li Shi, Chen Zhangyou, Zhang Lan, et al. Design of analog front-end for multi-channel dual-frequency $\mathrm{HF}$ radar receiver [J]. Application of electronic technology, 2018, 44(3): 31-35.

6. Deng Qianlan, Lu Zhongjie, Xinkang. Research on Calibration Technology of target echo simulator for pulse Doppler radar seeker [J]. Aerospace measurement technology, 2018, 38(1): 27-31. 